\title{
Using two soft computing methods to predict wall and bed shear stress in smooth rectangular channels
}

\author{
Zohreh Sheikh Khozani $^{1} \cdot$ Hossein Bonakdari ${ }^{1} \cdot$ Amir Hossein Zaji $^{1}$
}

Received: 26 July 2016/ Accepted: 21 February 2017/Published online: 3 March 2017

(c) The Author(s) 2017. This article is published with open access at Springerlink.com

\begin{abstract}
Two soft computing methods were extended in order to predict the mean wall and bed shear stress in open channels. The genetic programming (GP) and Genetic Algorithm Artificial Neural Network (GAA) were investigated to determine the accuracy of these models in estimating wall and bed shear stress. The GP and GAA model results were compared in terms of testing dataset in order to find the best model. In modeling both bed and wall shear stress, the GP model performed better with RMSE of 0.0264 and 0.0185 , respectively. Then both proposed models were compared with equations for rectangular open channels, trapezoidal channels and ducts. According to the results, the proposed models performed the best in predicting wall and bed shear stress in smooth rectangular channels. The obtained equation for rectangular channels could estimate values closer to experimental data, but the equations for ducts had poor, inaccurate results in predicting wall and bed shear stress. The equation presented for trapezoidal channels did not have acceptable accuracy in predicting wall and bed shear stress either.
\end{abstract}

Keywords Shear stress $\cdot$ Rectangular channel - Genetic Algorithm $\cdot$ Artificial neural network

Hossein Bonakdari

bonakdari@yahoo.com

1 Department of Civil Engineering, Razi University, Kermanshah, Iran

\section{Introduction}

Awareness of shear stress values along the wetted perimeter of channels is very important in solving engineering problems such as sedimentation and deposition also planning stable channels. To study channel migration or to prevent bank erosion, awareness of wall shear stress is required. The effective parameters in shear stress values are the channel geometry, secondary flows and the channel wall and bed roughness recognized through numerous experimental studies (Knight 1981; Tominaga et al. 1989; Knight and Sterling 2000; Atabay et al. 2004; Seckin et al. 2006). Based the division channel into two subsections, the channel bed and sidewall, by Einstein (1942), many researchers used this idea for estimating bed and wall shear stress, e.g., Lundgren and Jonsson (1964), Yang and Lim (2005), Khodashenas and Paquier (2002) and Yang (2005). The shear stress distribution in circular and trapezoidal channels was investigated using Shannon entropy-based power law by Sheikh and Bonakdari (2015). Bonakdari et al. (2015a, b) presented a new method of estimating the bed and wall shear stress along a wetted perimeter in open channels based on Tsallis entropy concept. The use of soft computing methods to solve complicated problems in hydraulic fields is expanding (Nagy et al. 2002; Cigizoglu 2004; Giustolisi and Laucelli 2005; Alp and Cigizoglu 2007). Cobaner et al. (2010) predicted the percentage of shear force in smooth rectangular channels and ducts using artificial neural networks (ANN). Huai et al. (2013) utilized the ANN method for estimating apparent shear stress in compound channels. The percentage of shear force carried by walls was modeled using genetic programming (GP) and Genetic Algorithm Artificial Neural Network (GAA) in rectangular channels 
with smooth and rough boundaries by Sheikh Khozani et al. $(2016 \mathrm{a}, \mathrm{b})$.

In this study, two different soft computing methods GP and GAA were extended in order to estimate mean wall and bed shear stresses in smooth rectangular channels. The two models were explained systematically, and in each stage, the best condition with the lowest error was selected after that the best GP or GAA model with highest precision was selected. In order to recognize the abilities of the proposed models, they were compared with equations presented by other researchers for rectangular channels, trapezoidal channels and ducts.

\section{Materials and methods}

\section{Data description used}

In order to predict the mean bed and wall shear stress in smooth rectangular channels, the experimental results of Cruff (1965), Ghosh and Roy (1970), Kartha and Leutheusser (1970), Myers (1978), Knight and Macdonald (1979), Knight (1981), Noutsopoulos and Hadjipanos (1982), Knight et al. (1984) and Seckin et al. (2006) were used. Different flumes with rectangular cross sections and several aspect ratios $(B / h)$ were employed in their experiments to estimate the mean bed shear stress and wall shear stress. Several equations were extracted from the researchers' results that can estimate shear stress in the bed and wall of a channel.

Knight (1981) expressed an equation for predicting wall shear stress in a rectangular channel as:

$\frac{\bar{\tau}_{w}}{\rho g h S_{f}}=\left(\frac{\% S F_{w}}{100}\right)\left(\frac{B}{2 h}\right)$,

$\frac{\bar{\tau}_{b}}{\rho g h S_{f}}=1-0.01 \% S F_{w}$,

where $\bar{\tau}_{w}$ is the mean wall shear stress, $\bar{\tau}_{b}$ the mean bed shear stress, $B$ the channel width, $h$ the water depth, $\rho$ the fluid density and $\% S F_{w}$ the total shear force carried by the walls calculated as:

$\% S F_{w}=e^{\alpha}\left(\tanh (\pi \beta)-0.5[\tanh (\pi \beta)-\beta]^{2}\right)$,

where

$\alpha=-3.264 \log \left(\frac{B}{h}+3\right)+6.211$

$\beta=1-\frac{\gamma}{5}$ and $\gamma=\log \left(\frac{k_{s b}}{k_{s w}}\right)$.

Using the concept of the relationship between $\% S F_{w}$ and the wetted perimeter ratio, $P_{b} / P_{w}$, Flintham and Carling
(1988) introduced a general equation for trapezoidal channels in the following form:

$\log \left(\% S F_{w}\right)=C_{1} \log \left(\frac{P_{b}}{P_{w}}+C_{2}\right)+C_{3}$,

where $C_{1}, C_{2}$ and $C_{3}$ are coefficients, and the limiting case is fixed by defining $\% S F_{w}=100 \%$ for $P_{b} / P_{w}=0$, then one constant is eliminated. Hence,

$C_{3}=2-C_{1} \log \left(C_{2}\right)$

and thus the transformed version of $\mathrm{Eq}$ (5) may be given by:

$\alpha=-3.23 \log \left(\frac{P_{b}}{P_{w}}+1\right)+4.6052$

The mean wall and bed shear stress can be estimated by:

$\frac{\bar{\tau}_{w}}{\rho g h S_{f}}=\left(\frac{\% S F_{w}}{100}\right)\left[\left(\frac{T+P_{b}}{4 h}\right) \sin \theta\right]$

$\frac{\bar{\tau}_{b}}{\rho g h S_{f}}=1-0.01 \% S F_{w}\left(\frac{T}{2 P_{b}}+0.5\right)$,

where $T$ is the width of the water surface and $\theta$ is the sidewall angle.Knight and Patel (1985) calculated boundary shear stress distributions in fully developed turbulent flows in smooth rectangular ducts and extracted the following equation:

$\% S F_{w}=\frac{103.23}{\left(1+\left(\frac{2 B}{3 h}\right)\right)^{1.4128}}$

Rhodes and Knight (1994) also proposed a blow-up equation for percentage of shear force in ducts:

$\% S F_{w}=\frac{100}{1+\left(\frac{1+1.345(h / B)}{1+1.345(B / h)}\right)^{-1.057}}$

The mean wall and bed shear stress was calculated using Eqs. (1) and (2).

\section{Genetic Algorithm-based artificial neural network}

The hybrid Genetic Algorithm (GA) and Multi-Layer Perceptron (MLP) artificial neural network were used in this study to calculate rectangular smooth channel wall and bed shear stresses. The MLP neural network is made from layers, which are input, hidden and output layers. The input layer receives input variables from the user and transfers them to the hidden layers. The hidden layer receives data from the input layer and after a nonlinear transformation, prepares appropriate information for the output layer. The output layer collects the previous layer's output and presents the MLP procedure results to the user. Each layer consists of some neurons. The input layer has the same 
number of neurons as the input variables of the model. In the present study, there is one input variable, $\mathrm{B} / \mathrm{H}$, so the input layer in the current MLP has only one neuron. The output layer has the same number of neurons as output variables of the model. General MLP models consider one output variable. Therefore, there is one neuron in the output layer.

Among the puzzling MLP modeling processes is determining the hidden layers' neuron number because there is no definitive rule for that. Therefore, in this study, a modified GA is employed in order to adjust the MLP structure by determining the number of neurons in the hidden layers. The hybrid GA-MLP method is demonstrated in Fig. 1. According to this figure, a random population of the MLP model with various numbers of hidden layer neurons is first generated, after which the modified GA generation begins in order to find the most appropriate hidden layer number of neurons. In the present MLP, two hidden layers are considered. Therefore, the modified GA has two outputs: one that represents the first hidden layer's number of neurons and another that represents the second hidden layer's number of neurons.

The Levenberg-Marquardt Algorithm (LMA) (Levenberg 1944) is used to train the considered MLP methods. The LMA has a random behavior in the determination of network weights and biases. Therefore, it is probable for a good MLP to be ignored in the GA process due to bad luck in the LMA procedure. As a result, modification is required in the GA optimization method. As shown in Fig. 1, in the modified GA, each individual (MLP) of the elite population is trained several times by the LMA to find the most appropriate result of the considered individual. After that, the best result of each individual is saved as its cost. By using this simple modification, the GA algorithm can successfully overcome the random nature of the LMA.

Another property of the MLP hidden layers that should be determined in the modeling process is transfer function selection. The sigmoid transfer function is often used as the transfer function of the hidden layers (Zadeh et al. 2010; Emiroglu et al. 2011; Pierini et al. 2012). The sigmoid function is defined as any function that is bounded and has a direct relation between the input variable and the output variable (Smith 1993). The linear, logarithmic and hyperbolic tangent transfer functions that are defined in Eqs. (13)-(15), respectively, are subsets of the sigmoid transfer function. In this study, the linear transfer function is used for the output layer, and the logarithmic and hyperbolic tangent activation functions are examined and compared on the hidden layers in order to find the most appropriate.

$$
\begin{aligned}
& \operatorname{purelin}(x)=x \\
& \log \operatorname{sig}(x)=\frac{1}{1+e^{-x}} \\
& \tan \operatorname{sig}(x)=\frac{2}{1+e^{-2 x}}-1
\end{aligned}
$$

\section{Genetic programming}

One of the most applicable subsets of the GA is Genetic Programming (GP) (Koza 1992). According to Fig. 2, this method undergoes the same procedure as the GA. However, GP uses the computer programs as individuals. Each computer program generated randomly could be a response

Fig. 1 The GAA flowchart

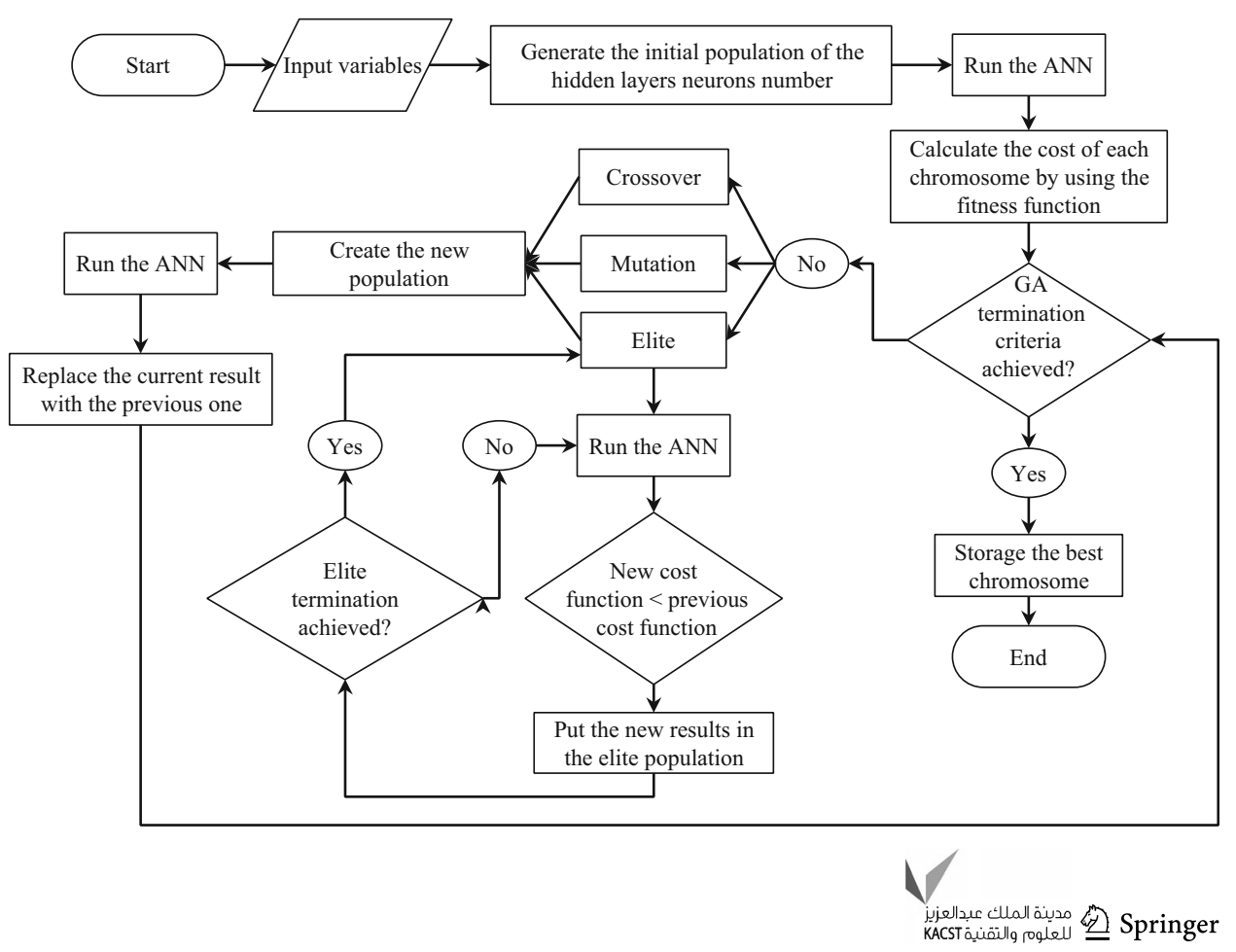


Fig. 2 The GP flowchart

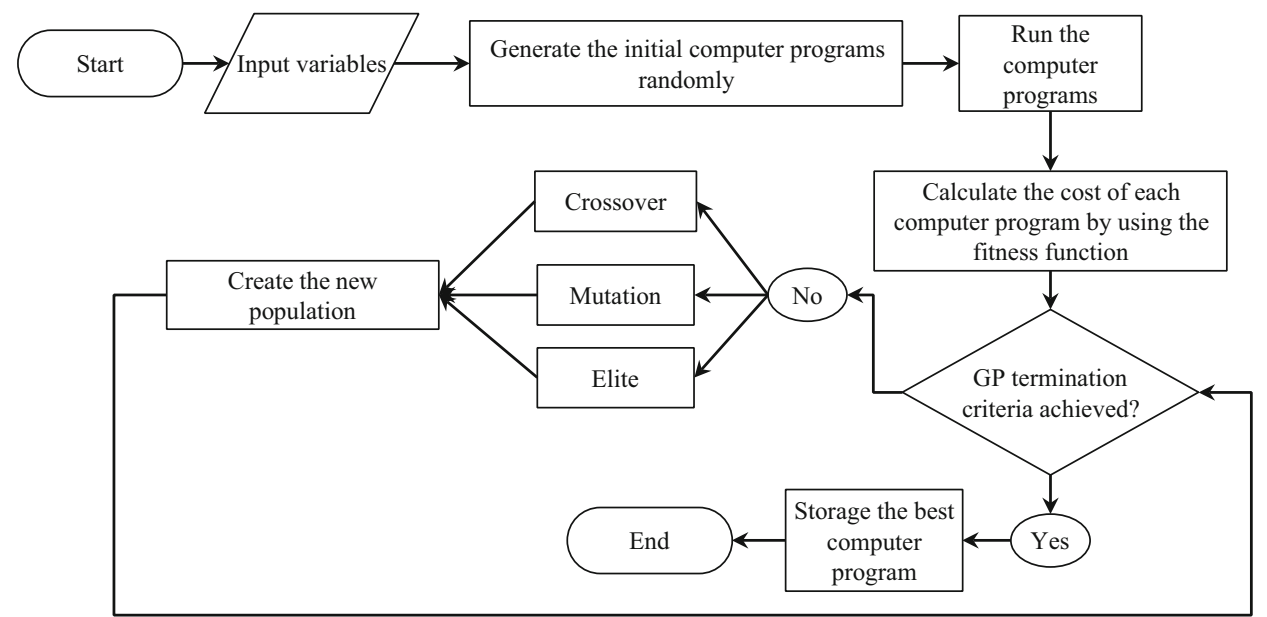

of the GP method. The computer program individuals are used to predict the considered model output. The GP starts with some random initial computer programs that serve as the initial population. The computer programs are randomly generated using the defined mathematical functions. The cost of each computer program is calculated with the fitness functions.

In the GP procedure, the computer programs are formed using some mathematical functions. Despite the high effect of appropriately selecting the computer programs' allowable functions there is no definitive rule on choosing them. Therefore, in this study, the function combinations of Eqs. (16)-(19) were considered. The GP was modeled using each function combination and the cost of each one is calculated. Function combination selection is based on the simplicity and complexity of the computer programs. Equations (16)-(19) demonstrate that the first function combination uses the simplest mathematical functions and by moving from the first function combination to the last, model complexity increases.

$F 1=(+,-, \times, \div)$

$F 2=(+,-, \times, \div, \sin , \cos )$

$F 3=(+,-, \times, \div$, sin, cos, abs, sqrt, power $)$

$F 4=(+,-, \times, \div$, sin, cos, abs, sqrt, power, exp $)$

In this study, the initial computer programs' length is considered 12 and their maximum length 64. Other properties of the GP considered are shown in Table 1.

\section{Model performance}

The performance of the GAA and GP models in predicting mean wall and bed shear stress were evaluated using statistical comparisons of the predicted and observed outputs. The comparison applied the three most commonly used error measures, RMSE, MAE and $\delta \%$. The root mean square error can be calculated by:

$\mathrm{RMSE}=\sqrt{\frac{\sum_{i=1}^{n}\left(x_{i p}-x_{i m}\right)^{2}}{n}}$,

where $x_{i p}$ is the mean wall or bed shear stress predicted by the models, $x_{i m}$ is the value of wall or bed shear stress obtained from the experimental results, and $n$ is the number of observations. Mean square error (MSE), MAE based on mean absolute error and average absolute deviation $(\delta \%)$ were also investigated. The results of comparing the models in each step as well as comparing the models with other methods were found and tabulated in different tables for each step. The other statistical parameters employed are defined as:

$\mathrm{MSE}=\frac{\sum_{i=1}^{n}\left(x_{i p}-x_{i m}\right)^{2}}{n}$

MAE $=\frac{1}{n} \sum_{i=1}^{n}\left|x_{i p}-x_{i m}\right|$

$\delta=\left(\frac{\sum_{i=1}^{n}\left|\left(x_{i p}-x_{i m}\right)\right|}{\sum_{i=1}^{n} x_{i p}}\right) \times 100$

\section{Results}

\section{Fitness function selection}

Considering the aspect ratio as input data, two different fitness functions were investigated to select the best one for modeling the mean wall and bed shear stress. The test dataset results are reported in Table 1. In modeling 
Table 1 GP properties

\begin{tabular}{ll}
\hline Parameter name & Parameter specification \\
\hline Population size & 500 \\
Mutation frequency & $93 \%$ \\
Crossover frequency & $50 \%$ \\
Number of replication & 10 \\
Block mutation rate & $30 \%$ \\
Instruction mutation rate & $30 \%$ \\
Instruction data mutation rate & $40 \%$ \\
Homologous crossover & $95 \%$ \\
\hline
\end{tabular}

dimensionless mean wall shear stress with the GAA model, MAE performed better with RMSE of 0.0305 than MSE with RMSE of 0.0402 . When the dimensionless mean wall shear stress was modeled with the GP model, the MSE showed better function than MAE did, with fitness functions of RMSE of 0.0276 and 0.0306 , respectively. It is obvious in Table 1 that in modeling $\frac{\bar{\tau}_{w}}{\rho g h S}$ the GP model performed better than the GAA model, with lower statistical parameter values.

The results of dimensionless mean bed shear stress modeling are shown is Table 2. A comparison of performance based on error statistics indicates that in the GAA model, MSE with $\delta \%=2.3751$ had better accuracy than the MAE fitness function with $\delta \%=2.6714$ for the testing dataset. The GP model with MAE as a fitness function presented lower error with RMSE of 0.0218 than the MSE fitness function with RMSE of 0.0276. Overall, in modeling $\frac{\bar{\tau}_{b}}{\rho g h S}$ the GP model with MAE fitness function had better accuracy than the GAA model with MSE fitness function.

\section{Selection of the best transfer function}

In the final step of GAA modeling, the appropriate transfer functions were investigated. Two commonly used types of sigmoid transfer functions are the logarithmic transfer function (Eq. 14) and hyperbolic tangent transfer function (Eq. 15). In this study, the logarithmic and hyperbolic tangent transfer functions were examined for the hidden layers and the linear transfer function (Eq. 13) was used for the output layer.

Table 3 shows the results of this stage for dimensionless wall or bed shear stress. As seen in this table, the best results of modeling $\frac{\bar{\tau}_{w}}{\rho g h S}$ were obtained by selecting the logarithmic transfer function in the hidden layer and purelin transfer function in the output layer. In fact, the logpur transfer function with MAE of 0.0240 performed better than the tan-pur transfer function with MAE of 0.0270. Considering the results in Table 3 for modeling $\frac{\bar{\tau}_{b}}{\rho g h S}$, it can be deducted that both log-pur and tan-pur transfer functions were highly accurate in GAA modeling in the last step. However, by selecting similar transfer functions for dimensionless wall shear stress (i.e., logarithmic transfer function in the hidden layer and purelin transfer function in the output layer), the model exhibits better performance. The difference between the results of log-pur and tan-pur transfer functions in modeling $\frac{\bar{t}_{b}}{\rho g h S}$ is very small (about 0.0001 RMSE) and indicates that both transfer functions are appropriate.

The output equation of the best GAA model with aspect ratio as input data, MAE as fitness function and log-pur as transfer function is:

$$
\begin{aligned}
\frac{\bar{\tau}_{w}}{\rho g h S}= & \operatorname{purelin}\left(\left(\operatorname { l o g } \operatorname { s i g } \left(\left(\log \operatorname{sig}\left(\frac{b}{h} \times\left[\begin{array}{c}
-0.11 \\
1.14
\end{array}\right]^{T}+\left[\begin{array}{c}
-11.23 \\
1.15
\end{array}\right]^{T}\right)\right)\right.\right.\right. \\
& \left.\left.\left.\times\left[\begin{array}{cc}
-3.46 & 9.07 \\
-6.91 & -5.96 \\
4.37 & -7.05
\end{array}\right]^{T}+\left[\begin{array}{l}
2.18 \\
6.83 \\
8.61
\end{array}\right]^{T}\right)\right) \times\left[\begin{array}{c}
1.37 \\
-1.74 \\
-1.46
\end{array}\right]+1.68\right)
\end{aligned}
$$

where purelin and logsig are Eqs. (13) and (14), respectively.

The output of the best GAA model in modeling bed shear stress with aspect ratio as input data, MSE as fitness function and log-pur is a very simple equation, as follows:

\begin{tabular}{|c|c|c|c|c|c|c|c|}
\hline \multirow[t]{2}{*}{ Fitness functions } & \multirow[t]{2}{*}{ Variables } & \multicolumn{3}{|l|}{ GAA } & \multicolumn{3}{|l|}{ GP } \\
\hline & & RMSE & MAE & $\delta \%$ & RMSE & MAE & $\delta \%$ \\
\hline \multirow[t]{2}{*}{ MSE } & $\frac{\bar{\tau}_{w}}{\rho g h S}$ & 0.0402 & 0.0324 & 5.6952 & 0.0276 & 0.0219 & 3.843 \\
\hline & $\frac{\bar{\tau}_{b}}{\rho g h S}$ & 0.0229 & 0.0173 & 2.3751 & 0.0231 & 0.0178 & 2.4058 \\
\hline \multirow[t]{2}{*}{ MAE } & $\frac{\bar{\tau}_{w}}{\rho g h S}$ & 0.0305 & 0.0240 & 4.2106 & 0.0306 & 0.0249 & 4.4187 \\
\hline & $\frac{\bar{\tau}_{b}}{\rho g h S}$ & 0.0258 & 0.0194 & 2.6714 & 0.0218 & 0.0151 & 2.0423 \\
\hline
\end{tabular}

Table 2 Measured errors for different fitness functions for the testing dataset 
Table 3 Statistical parameter values of transfer function selection for the testing dataset

\begin{tabular}{|c|c|c|c|c|c|c|c|}
\hline \multirow[t]{2}{*}{ Hidden layer transfer function } & \multirow[t]{2}{*}{ Output layer transfer function } & \multicolumn{3}{|l|}{$\frac{\bar{\tau}_{W}}{\rho g h S}$} & \multicolumn{3}{|l|}{$\frac{\bar{\tau}_{b}}{\rho g h S}$} \\
\hline & & RMSE & MAE & $\delta \%$ & RMSE & MAE & $\% \delta$ \\
\hline Logsig & Purelin & 0.0305 & 0.0240 & 4.2106 & 0.0229 & 0.0173 & 2.3751 \\
\hline Lansig & Purelin & 0.0347 & 0.0270 & 4.7316 & 0.0230 & 0.0174 & 2.3926 \\
\hline
\end{tabular}

$$
\begin{aligned}
\frac{\bar{\tau}_{b}}{\rho g h S}= & \operatorname{pure}\left(\left(\operatorname { l o g } s \left(\left(\log s\left(\frac{b}{h} \times(-0.17)-2.47\right)\right)\right.\right.\right. \\
& \times(-28.24)+4.9)) \times 16.14-15.08)
\end{aligned}
$$

Evidently, the GAA model can present explicit and simple equations for predicting wall and bed shear stress, whereas several algorithm genetic methods cannot present equations as output.

\section{Selection of the best mathematical function set}

In this study, different mathematical functions were used in the final step of GP modeling, including basic arithmetic operators $(+,-, \times, \div)$ as well as some other basic mathematical functions (sin, cos, abs, sqrt, power, exp). The different combinations of mathematical functions were investigated as per Eqs. (16)-(19).

The selection of appropriate mathematical functions has high impact on model performance. The results of the comparison are tabulated in Table 4. In modeling wall shear stress, the $F 2$ mathematical function with the lowest error had the best accuracy among the other function sets. With increasing the depended functions the error values increased. As seen in Table 4, modeling with $F 3$ resulted in RMSE of 0.0316 that indicates the worst accuracy.

In modeling bed shear stress, the combination of all mathematical functions used, F4, with RMSE of 0.0185 was the most appropriate among the basic functions, while $F 1$ was second best. It is obvious that there are no basic rules for identifying which has a better function than the rest and the linking function selection depends on the problem. Both parameters modeled with $F 3$ had the highest errors.

The more appropriate GP model's output in modeling the mean wall shear stress with aspect ratios as input data, MSE as fitness function and $F 2$ as mathematical function set is displayed as the program in Table 5. The output program for modeling mean bed shear stress in the GP model with aspect ratios as input data, MAE as fitness function and $F 4$ as mathematical function set is presented in Table 5. These programs were written in Matlab software, and in these programs $\mathrm{Tw}$ is $\frac{\bar{\tau}_{w}}{\rho g h S}$ and $\mathrm{Tb}$ is $\frac{\bar{\tau}_{b}}{\rho g h S}$.

\section{Selection of the more appropriate model}

At this stage, a comparison between the GAA and GP models was done in order to select the more appropriate model for predicting wall and bed shear stresses. The obtained results are shown in Table 6. It is clear that both parameters modeled with GP performed better than with the GAA model. In modeling $\frac{\bar{\tau}_{w}}{\rho g h S}$, the GP with MAE of 0.0219 had better function than the GAA model with MAE of 0.0240. Therefore, the GP model was selected as the model with higher performance in predicting wall shear stress. Since in modeling $\frac{\bar{\tau}_{b}}{\rho g h S}$ with the proposed models GP with RMSE of 0.0185 was more accurate than GAA with RMSE of 0.0229 , the GP model was chosen for predicting bed shear stress.

\section{Comparison between the best model and other equations}

In order to investigate the proposed model's ability to predict shear stress along the wall and the bed of a smooth rectangular channel, this model was compared with other formulae presented by different researchers. The comparison results for different methods of predicting wall shear stress for the entire dataset are shown in Table 7. The proposed models with the lowest statistical parameter values, RMSE of 0.04637 and 0.0486 for the GAA and GP models, respectively, performed the best compared to the others. The equation for rectangular channels presented by Knight (1981) could predict reasonable wall shear stress values, since its statistical parameter values are close to the proposed models. The presented equation for a trapezoidal channel could not predict acceptable values for wall shear stress, and with RMSE of 0.1826 , it exhibited low precision for predicting wall shear stress. The equations proposed for smooth ducts presented by Knight and Patel (1985) and Rhodes and Knight (1994) had the highest error values (RMSE of 0.2694 and 0.2554 , respectively) and indicated the equations' poor accuracy in predicting wall shear stress.

The results of the comparison between the GAA and GP models with other equations obtained by researchers are also illustrated in Fig. 3. If the $R^{2}$ value is close to 1 the 
Table 4 Preliminary selection of mathematical functions in the GP model for the testing dataset

\begin{tabular}{|c|c|c|c|c|c|c|}
\hline \multirow[t]{2}{*}{ Mathematical functions } & \multicolumn{3}{|l|}{$\frac{\bar{\tau}_{w}}{\rho g h S}$} & \multicolumn{3}{|l|}{$\frac{\bar{\tau}_{b}}{\rho g h S}$} \\
\hline & RMSE & MAE & $\delta \%$ & RMSE & MAE & $\delta \%$ \\
\hline$F 1$ & 0.0276 & 0.0219 & 3.843 & 0.0218 & 0.0151 & 2.0423 \\
\hline$F 2$ & 0.0264 & 0.0219 & 3.8153 & 0.0223 & 0.0134 & 1.8227 \\
\hline$F 3$ & 0.0316 & 0.0263 & 4.6265 & 0.0229 & 0.0148 & 2.0090 \\
\hline$F 4$ & 0.0273 & 0.0228 & 3.9497 & 0.0185 & 0.0110 & 1.4866 \\
\hline
\end{tabular}

Table 5 The output program of the GP model for (a) modeling the mean wall shear stress and (b) modeling the mean bed shear stress (a)

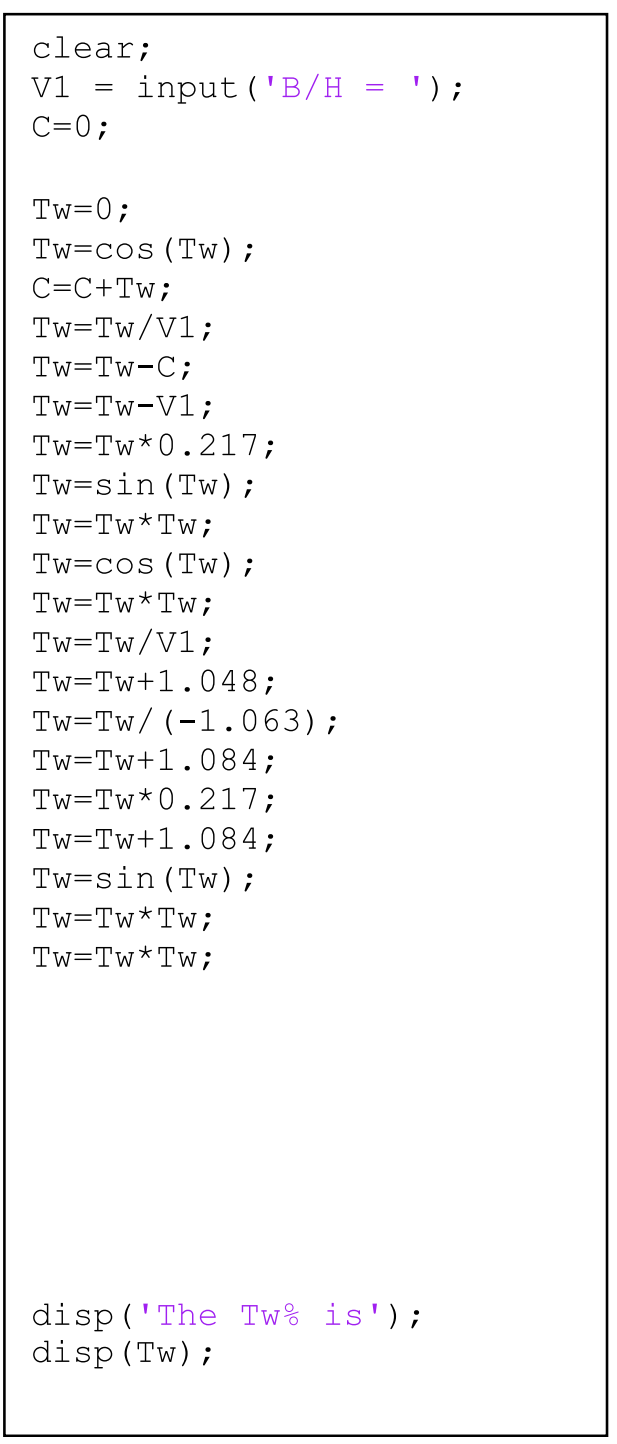

(b)

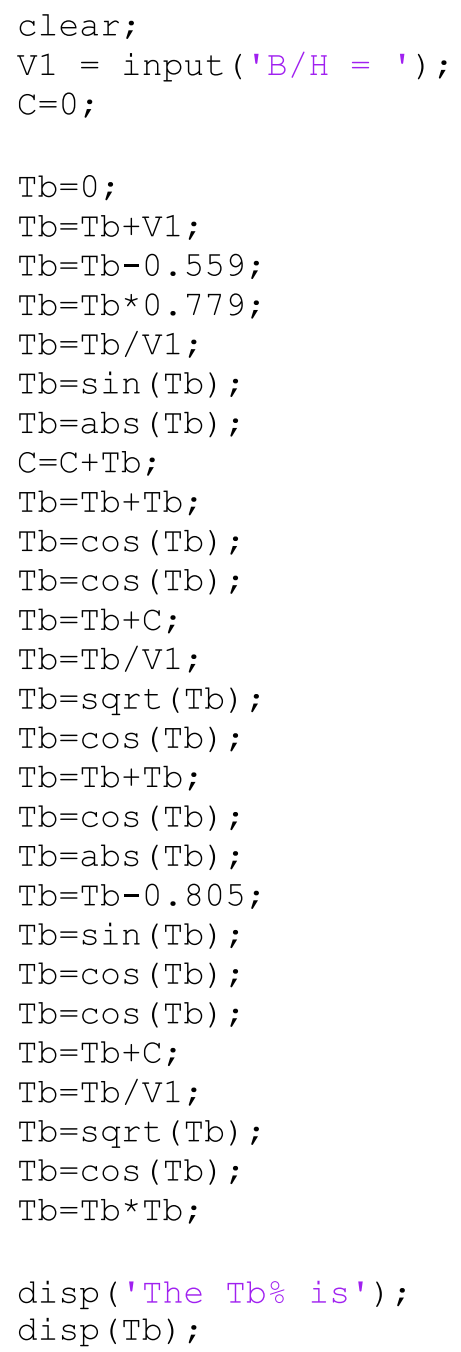

best fit of observed versus predicted values is achieved. The proposed model results are close to the fitted line since the $R^{2}$ value is 0.8775 for the GAA model and 0.8671 for the GP model. Knight's (1981) equation is better than the equations for duct and trapezoidal channels with respect to $R^{2}$. As seen in Fig. 3, Flintham and Carling's (1988) equation estimates lower values for wall shear stress and it can be deducted that the equation for trapezoidal channels 
Table 6 Comparison between GAA and GP models

\begin{tabular}{|c|c|c|c|c|c|c|}
\hline \multirow[t]{2}{*}{ Variables } & \multicolumn{3}{|l|}{ GAA } & \multicolumn{3}{|l|}{ GP } \\
\hline & RMSE & MAE & $\delta \%$ & RMSE & MAE & $\delta \%$ \\
\hline$\frac{\bar{\tau}_{w}}{\rho g h S}$ & 0.0305 & 0.0240 & 4.2106 & 0.0264 & 0.0219 & 3.8153 \\
\hline$\frac{\bar{\tau}_{b}}{\rho g h S}$ & 0.0229 & 0.0173 & 2.3751 & 0.0185 & 0.0110 & 1.4866 \\
\hline
\end{tabular}

Table 7 Comparison between GAA and GP models with other equations for wall shear stress

\begin{tabular}{lllr}
\hline Methods & \multicolumn{2}{l}{ Statistical parameters } & MAE \\
\cline { 2 - 4 } & RMSE & 0.03202 & 5.9596 \\
\hline GAA model & 0.0463 & 0.0358 & 6.6082 \\
GP model & 0.0486 & 0.0429 & 7.8096 \\
Presented equation by Knight (1981) & 0.0543 & 0.1645 & 44.0806 \\
Presented equation by Flintham and Carling (1988) & 0.1826 & 0.2483 & 85.8381 \\
Presented equation by Knight and Patel (1985) & 0.2694 & 0.2383 & 79.6243 \\
Presented equation by Rhodes and Knight (1994) & 0.2554 & & \\
\hline
\end{tabular}

is not useful for calculating wall shear stress in rectangular channels. The values predicted by the duct equations are underestimated and these equations had better not be used for estimating wall shear stress in rectangular channels. As seen in Fig. 3, the equations proposed by Flintham and Carling's (1988), Rhodes and Knight (1994) and Knight and Patel (1985) predicted underestimated values for mean wall shear stress that resulted in designing channels with high erosion. Since the actual values of wall shear stress are higher than the values predicted by these equations, wall protective structures are needed; while designing channel with wall shear stress obtained by GP and GAA resulted in more stable channels.

The bed shear stress values predicted by the proposed models and equations were compared and the results are tabulated in Table 8. According to the results, the GAA model with RMSE of 0.0293 and the GP model with RMSE of 0.0312 have greater ability to predict bed shear stress. The results of the equation presented by Knight (1981) are similar to the proposed models' results. Equation (10) with RMSE of 0.0951 had lower accuracy than the proposed model and equation obtained for rectangular channels. Considering the results in Tables 7 and 8, the results of Eq (10) are more accurate than Eq (9). The duct equations presented the worst outcome in predicting bed shear stress, with RMSE of 0.1423 and 0.1458 , respectively. Overall, Tables 7 and 8 signify that all methods perform better in predicting bed shear stress than wall shear stress.

Figure 4 shows the results of the comparison between the proposed models and other equations for bed shear stress in the form of a scatter plot. Both proposed models could predict bed shear stress values very close to experimental data. In addition, the equation obtained for rectangular channels could estimate values close to the fitted line. Flintham and Carling's (1988) equation for trapezoidal channels calculated overestimated values for bed shear stress and the impact of existing secondary flows can be effective on overestimating values. It obvious from Fig. 4 that the relationships for estimating bed shear stress in ducts, are not appropriate for predicting bed shear stress in open channels. As seen from the fit line equations in ducts (assuming the equation is $y=a_{1} x+a_{2}$ ), in the scatterplots the $a_{2}$ coefficients have very high values: 0.2643 for Knight and Patel's (1985) equation and 0.2749 for Rhodes and Knight's (1994) equation. As seen in Fig. 4, the obtained equations for ducts could not predict accurate values for bed shear stress in open channels. Since the presented equations in trapezoidal channels and ducts predicted overestimated values of bed shear stress, the designed channels by these values resulted in high construction costs. The designed channels based the bed shear stress values obtained by GP, GAA and equation proposed by Knight (1981) due to design stable and affordable channels.

\section{Conclusion}

Because shear stress plays an important role in hydraulic problems of channels two soft computing methods, GAA and GP models were developed to estimate wall and bed shear stress in smooth rectangular channels. For GP 
Fig. 3 Comparison between proposed models and other equations for wall shear stress
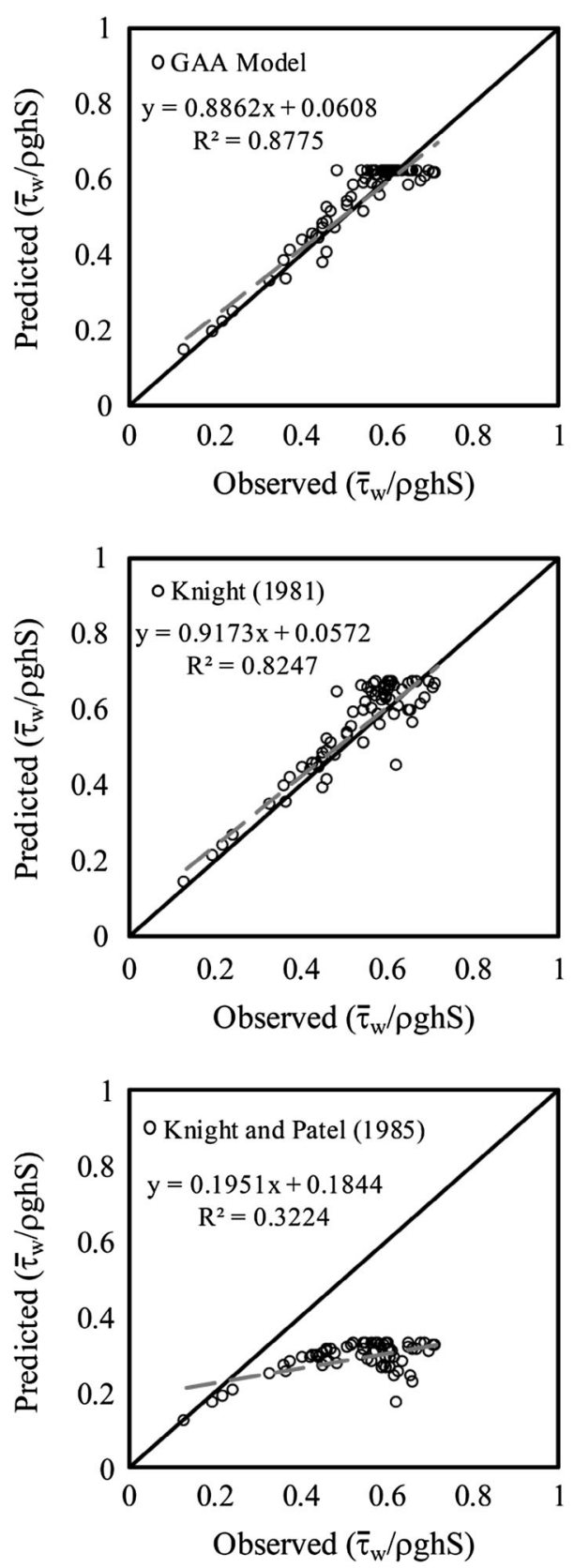
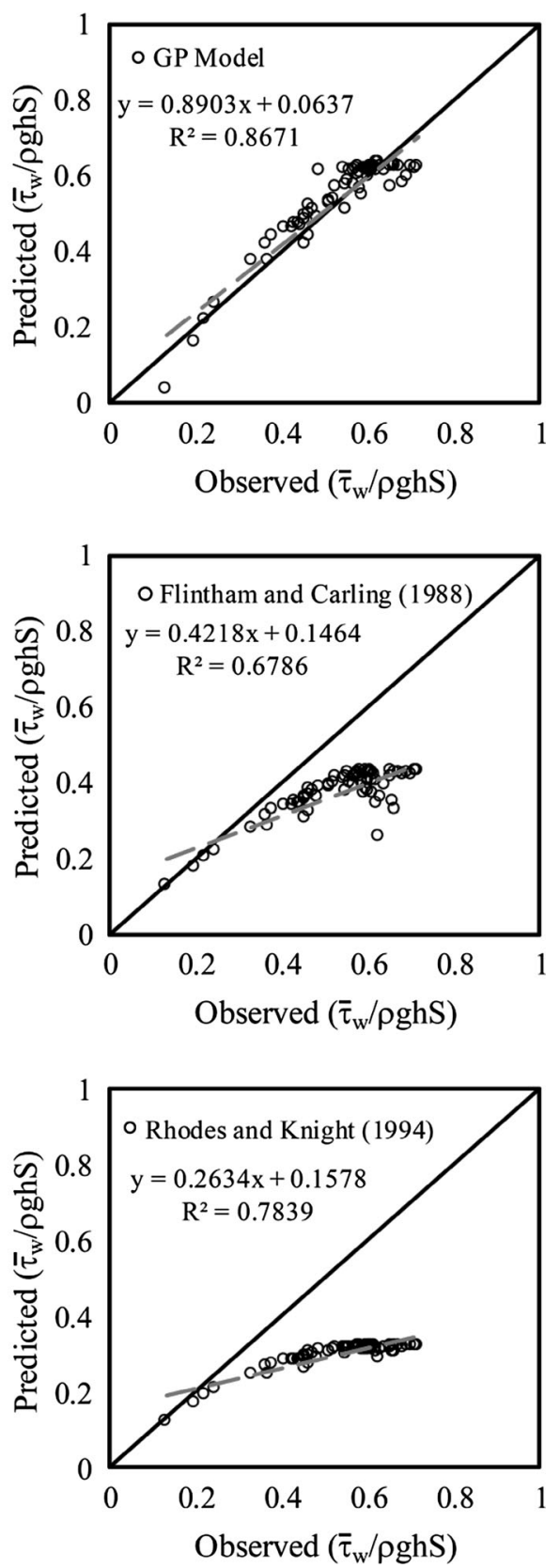

Table 8 Comparison between GAA and GP models with other equations for mean bed shear stress

\begin{tabular}{llrr}
\hline Methods & \multicolumn{2}{l}{ Statistical parameters } & \multicolumn{2}{l}{ MAE } & \multicolumn{2}{l}{$\delta \%$} \\
\cline { 2 - 4 } & RMSE & 0.0209 & 3.2288 \\
\hline GAA model & 0.0293 & 0.0183 & 2.7894 \\
GP model & 0.0312 & 0.0242 & 3.7667 \\
Presented equation by Knight (1981) & 0.0318 & 0.0830 & 11.3135 \\
Presented equation by Flintham and Carling (1988) & 0.0951 & 0.1273 & 16.2633 \\
Presented equation by Knight and Patel (1985) & 0.1423 & 0.1284 & 16.4748 \\
Presented equation by Rhodes and Knight (1994) & 0.1458 & \\
\hline
\end{tabular}


Fig. 4 Comparison between proposed models and other equations for bed shear stress
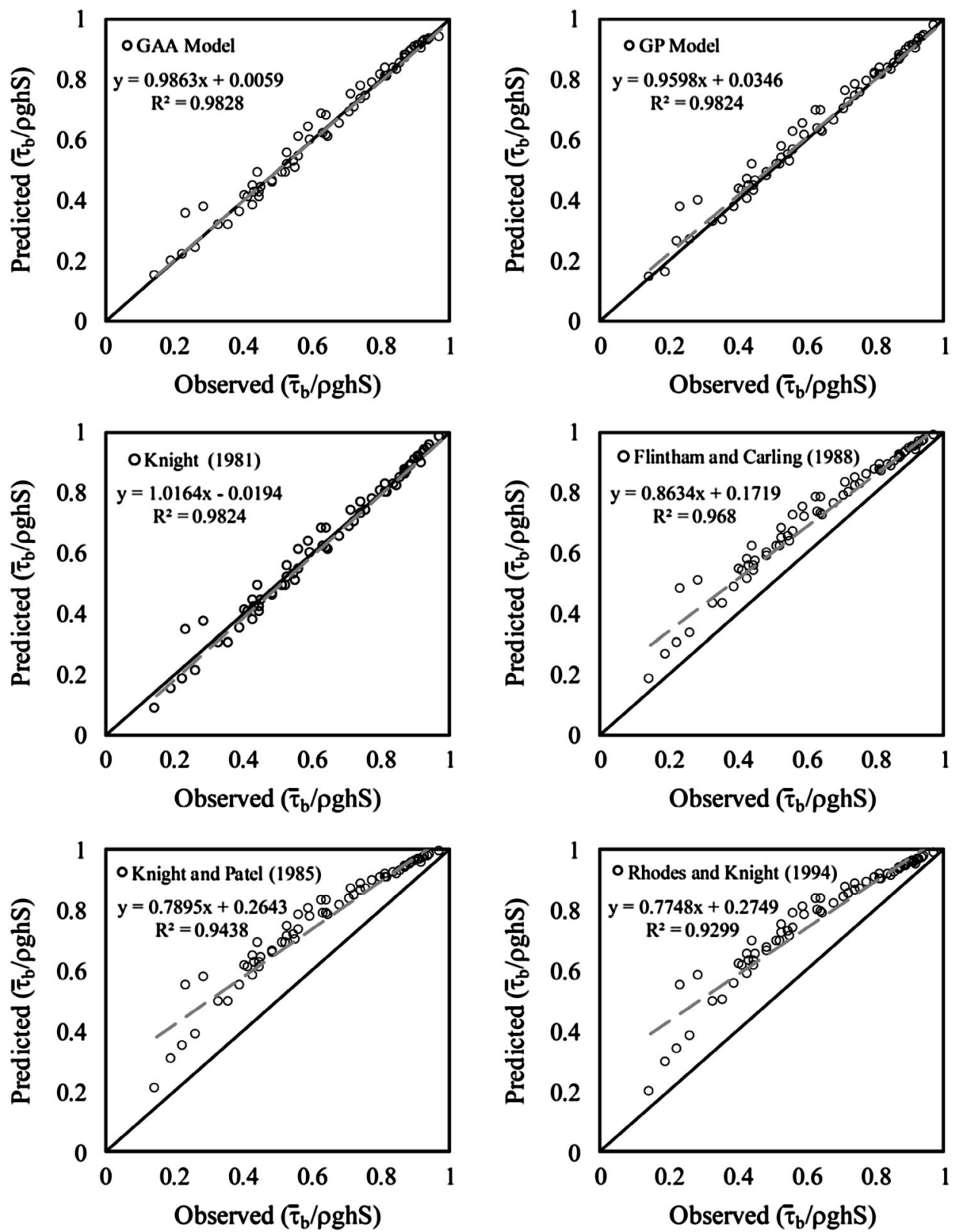

modeling, several fitness functions and mathematical functions were investigated and the most appropriate was selected to find the best GP model. According to the results for modeling mean wall shear stress MSE as fitness function and $F 2$ as mathematical function indicated the best results with RMSE of 0.0262. Also for mean bed shear stress MAE as fitness function and $F 4$ as mathematical function showed the best results with RMSE of 0.0185 than other GP models. For GAA modeling, different fitness functions and transfer functions were studied and the best was chosen to make the most appropriate GAA model. Both proposed models were compared with each other with the testing dataset based on statistical parameter values and the model with higher accuracy was introduced. The results showed that both GAA and GP models were highly accurate in predicting bed and wall shear stress. These models' results were then compared with an equation for rectangular channels presented by Knight (1981), an equation for trapezoidal channels presented by Flintham and Carling (1988) and two equations by Knight and Patel (1985) and Rhodes and Knight (1994) for rectangular ducts for the entire dataset. These results were obtained as:

1. Considering the results, the GAA and GP models exhibited greater ability than the other equations, with RMSE of 0.0463 and 0.0486 , respectively, for wall shear stress modeling and RMSE of 0.0293 for the GAA model and 0.0312 for the GP model for bed shear stress. 
2. After the proposed models, the equation for rectangular channels proposed by Knight (1981) had reasonable precision in predicting wall shear stress.

3. For bed shear stress, Flintham and Carling's (1988) equation predicted underestimated values and had high errors similar to the wall shear stress results. The equation for trapezoidal sections could not predict acceptable values for wall shear stress and all predicted values were underestimated.

4. The equations for rectangular ducts had the worst accuracy in predicting wall and bed shear stress. In estimating wall shear stress, the results obtained with the equations for ducts were underestimated and for estimating bed shear stress, they were overestimated.

5. Generally, soft computing methods can be powerful means of predicting shear stress in rectangular channels and their results can result in designing more stable and affordable channels.

Open Access This article is distributed under the terms of the Creative Commons Attribution 4.0 International License (http:// creativecommons.org/licenses/by/4.0/), which permits unrestricted use, distribution, and reproduction in any medium, provided you give appropriate credit to the original author(s) and the source, provide a link to the Creative Commons license, and indicate if changes were made.

\section{References}

Alp M, Cigizoglu HK (2007) Suspended sediment load simulation by two artificial neural network methods using hydro meteorological data. Environ Model Softw 22:2-13

Atabay S, Knight DW, Seckin G (2004) Influence of a mobile bed on the boundary shear in a compound channel. In Proc Int Conf River Flow,Napoli, Italy, vol. 1, pp. 337-345

Bonakdari H, Sheikh Z, Tooshmalani M (2015a) Comparison between Shannon and Tsallis entropies for prediction of shear stress distribution in circular open channels. Stoch Env Res Risk Assess 29(1):1-11

Bonakdari H, Tooshmalani M, Sheikh Z (2015b) Predicting shear stress distribution in rectangular channels using entropy concept. Int J Eng 28(3):360-367

Cigizoglu HK (2004) Estimation and forecasting of daily suspended sediment data by multi layer perceptrons. Adv Water Resour 27:185-195

Cobaner M, Seckin G, Seckin N, Yurtal R (2010) Boundary shear stress analysis in smooth rectangular channels and ducts using neural networks. Water Environ J 24:133-139

Cruff RW (1965) Cross-channel transfer of linear momentum in smooth rectangular channels. Geological Survey Water Supply Paper 1592-B, US Geological Survey, Washington, DC, pp. B1-B26

Einstein HA (1942) Formulas for the transportation of bed-load. Trans ASCE 107(2140):561-597

Emiroglu ME, Bilhan O, Kisi O (2011) Neural networks for estimation of discharge capacity of triangular labyrinth sideweir located on a straight channel. Expert Sys Appl 38:867-874

Flintham TP, Carling PA (1988) The prediction of mean bed and wall boundary shear in uniform and compositely rough channels. Proc. Int. Conf. River Regime, Wiley, Chichester, pp. 267-287
Ghosh SN, Roy N (1970) Boundary shear distribution in open channel flow. J Hydraul Div 96(4):967-994

Giustolisi O, Laucelli D (2005) Improving generalization of artificial neural networks in rainfall-runoff modelling. Hydrolog Sci 50(3):439-457

Huai W, Chen G, Zeng Y (2013) Predicting apparent shear stress in prismatic compound channels using artificial neural network. J Hydroinform 15(1):131-146

Kartha VC, Leutheusser HJ (1970) Distribution of tractive force in open channels. J Hydraul Div 96(7):1469-1483

Khodashenas SR, Paquier A (2002) River bed deformation calculated from boundary shear stress. J Hydraul Res 40(5):603-609

Knight DW (1981) Boundary shear in smooth and rough channels. J Hydraul Div 107(7):839-851

Knight DW, Macdonald JA (1979) Open channel flow with varying bed roughness. J Hydraul Div 105(9):1167-1183

Knight DW, Patel HS (1985) Boundary shear stress in smooth rectangular ducts. J Hydraul Div 111(1):29-47

Knight DW, Sterling M (2000) Boundary shear in circular pipes partially full. J Hydraul Eng 126(4):263-275

Knight DW, Demetriou JD, Hamed ME (1984) Boundary shear stress in smooth rectangular channel. J Hydraul Eng 10(4):405-422

Koza JR (1992) Genetic programming: on the programming of computers by means of natural selection. MIT press, Cambridge

Levenberg K (1944) A method for the solution of certain non-linear problems in Least-Squares. Qu Appl Math 2:164-168

Lundgren H, Jonsson IG (1964) Shear and velocity distribution in shallow channel. J Hydraul Div 90(1):1-21

Myers WRC (1978) Momentum transfer in a compound channel. J Hydraul Res 16(2):139-150

Nagy HM, Watanabe K, Hirano M (2002) Estimation of sediment load concentration in rivers using artificial neural network model. J Hydraul Eng 128(6):588-595

Noutsopoulos GC, Hadjipanos PA (1982) Discussion of "boundary shear in smooth and rough channels"' by D.W. Knight. J Hydraul Eng 108(6):809-812

Pierini JO, Gomez EA, Telesca L (2012) Prediction of water flows in Colorado River, Argentina. Lat Am J Aquat Res 40:872-880

Rhodes DG, Knight DW (1994) Distribution of shear force on boundary of smooth rectangular duct. J Hydraul Eng 120(7):787-807

Seckin G, Seckin N, Yurtal R (2006) Boundary shear stress analysis in smooth rectangular channels. NRC Res Press 33:336-342

Sheikh Z, Bonakdari H (2015) Prediction of boundary shear stress in circular and trapezoidal channels with entropy concept. J Urban Water 13(6):629-636

Sheikh Khozani Z, Bonakdari H, Zaji AM (2016a) Application of a genetic algorithm in predicting the percentage of shear force carried by walls in smooth rectangular channels. Measurement 87:87-98

Sheikh Khozani Z, Bonakdari H, Zaji AM (2016b) Application of soft computing technique in prediction percentage of shear force carried by walls in rectangular channel with Non-homogenous roughness. Water Sci Technol 73(1):124-129

Smith M (1993) Neural networks for statistical modeling. Thomson Learning, Boston

Tominaga A, Nezu I, Ezaki K, Nakagawa H (1989) Three dimensional turbulent structure in straight open channel flows. J Hydraul Res 27:149-173

Yang SQ (2005) Interaction of boundary shear stress, secondary currents and velocity. Fluid Dyn Res 36(3):121-136

Yang SQ, Lim SY (2005) Boundary shear stress distributions in trapezoidal channels. J Hydraul Res 43(1):98-102

Zadeh MR, Amin S, Khalili D, Singh VP (2010) Daily outflow prediction by multi layers perceptron with logistic sigmoid and tangent sigmoid activation functions. Water Resour Manag 24:2673-2688

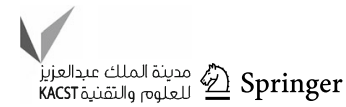

